\title{
WEAKLY AMENABLE GROUPS AND AMALGAMATED PRODUCTS
}

\author{
MAREK BOŻEJKO AND MASSIMO A. PICARDELLO
}

(Communicated by Jonathan M. Rosenberg)

\begin{abstract}
Denote by $B_{2}(G)$ the Herz-Schur multiplier algebra of a locally compact group $G$ and by $B_{2, \lambda}(G)$ the closure of the Fourier algebra in the topology of pointwise convergence boundedly in the norm of $B_{2}(G) . G$ is said to be weakly amenable if $B_{2, \lambda}(G)=B_{2}(G)$. We show that every amalgamated product of a countable collection of locally compact amenable groups over a compact open subgroup is weakly amenable. This improves and extends previous results that hold for amalgams of compact groups.
\end{abstract}

\section{INTRODUCTION}

Let $G$ be a locally compact group, $A(G)$ its Fourier algebra and $B(G)$ its Fourier-Stieltjes algebra. Denote by $B_{\lambda}(G)$ the closure of $A(G)$ in the topology of uniform convergence on compact sets, boundedly in norm. Then $G$ is amenable if and only if $B_{\lambda}(G)=B(G)$. Moreover, if $G$ is amenable, then $B(G)=\mathscr{M} A(G)$ (the algebra of multipliers of $A(G)$ ).

Recent papers on amenability have devoted a significant amount of attention to the Herz-Schur multiplier algebra $B_{2}(G)$ [He]. It was noted in [BF] that $B_{2}(G)$ coincides with the algebra $\mathscr{M}_{0} A(G)$ of completely bounded multipliers of $A(G)$, introduced in [dCH], where many of its interesting properties were investigated. Amenability can be characterized in terms of $B_{2}(G)$ as follows. $G$ is amenable if and only if $B(G)=B_{2}(G)$ ([Lo]; for discrete groups, [Bo]). Actually, for the purpose of studying amenability, the multiplier algebra $B_{2}(G)$ is better suited than $\mathscr{M} A$, because it has nicer functorial properties. For instance, the Herz-Schur multiplier constant $\Lambda_{G}$ (i.e., the infimum of the norms of all approximate identities in $B_{2}(G)$ ) is a von Neumann algebra invariant ([Ha2]; see $[\mathrm{CoH}]$ for a deep study of this invariant on simple Lie groups of real rank 1), whereas a similar statement does not hold for $\mathscr{M} A$. Above all, $B_{2}(G)$ is nicer in taking products. In fact, $B_{2}\left(G_{1}\right) \otimes B_{2}\left(G_{2}\right) \subseteq B_{2}\left(G_{1} \times G_{2}\right)$ [dCH], but the same statement does not hold for $\mathscr{M} A$. This paper makes use of $B_{2}(G)$ to study a property related to amalgamation for another type of products: free products with amalgamation. This property, studied in $\S 2$, can be naturally phrased in

Received by the editors July 23, 1990 and, in revised form, July 19, 1991.

1991 Mathematics Subject Classification. Primary 43A35, 05C05; Secondary 05C25.

Key words and phrases. Herz-Schur multipliers, weakly amenable groups, topological groups with amalgamation, homogeneous and semihomogeneous trees, positive definite functions. 
terms of the Herz-Schur algebra as follows. Denote by $B_{2, \lambda}(G)$ the closure of $A(G)$ in the topology of uniform convergence on compact sets, boundedly in the $B_{2}$-norm. $G$ is called weakly amenable if and only if $B_{2, \lambda}(G)=B_{2}(G)$.

We focus attention on amalgamated products $G={ }_{*} G_{i}$, where the $G_{i}$ 's are locally compact amenable groups and $A$ is a compact open subgroup in each $G_{i}$. It was shown in [Pi], under the assumption that the indices $\left[G_{i}: A\right]$ are bounded, that there exist (unbounded) approximate identities in $A(G)$ that are bounded in the norm of $\mathscr{M} A(G)$. Here we show more (Theorem 4.2). There exist approximate identities bounded in $B_{2}(G)$. That is, $G$ is weakly amenable. Moreover, we drop the assumption that $\left[G_{i}: A\right]$ be bounded, which is very restrictive, because it forces all the $G_{i}$ 's to be compact. The approach of [Pi] (see also [FP1]) is based upon Haagerup's estimates for convolution operators [Ha1] and the fact that the exponential function $g \rightarrow r\|g\|$ is positive definite (here $\|g\|$ is the length function on $G$ and $0<r \leq 1$ ). These estimates are related to the geometry of trees, more precisely, to the uniqueness of the representation of elements of $G$ as words in the $G_{i}$ 's ([Se]; see Theorem 3.1 below). In the environment of trees, the desired estimates reduce to Haagerup's bound for the multiplier norm of the characteristic function $\chi_{n}$ of words of length $n$ [Hal]. Our proof is based upon similar bounds for $\chi_{n}$ (in the $B_{2}$ norm). The desired bounds are first obtained on trees ( $(2)$, and then carried through to amalgams, by making use of Chiswell's length function $(\S 3)$. We emphasize the fact that all our arguments are base solely upon the geometry of trees and groups. To our knowledge, all previous papers concerning HerzSchur multipliers and weakly amenable groups made use of a more abstract tool (although interesting per se), namely, uniformly bounded representations on Hilbert spaces. In particular, results partially ovęrlapping ours were proved by this token in [Sz].

Unexplained terminology and notation are as in [Di, FP1, Pi, Se].

\section{WeAKly AMENABle GROUPS AND HERZ-SCHUR MULTIPLIERS ON TREES}

Let $X$ be an arbitrary set, and denote by $V_{2}(X)$ be the set of Schur multipliers on $X:$ a function $a: X \times X \rightarrow \mathbb{C}$ belongs to $V_{2}(X)$ if there exist maps $\alpha$ and $\beta$ from $X$ to a Hilbert space $\mathscr{H}$ such that

$$
\begin{gathered}
\sup _{x}\|\alpha(x)\|=C_{1}<\infty, \quad \sup _{x}\|\beta(x)\|=C_{2}<\infty, \\
a(x, y)=\langle\alpha(x), \beta(y)\rangle \quad \text { for } x, y \in X .
\end{gathered}
$$

The algebra $V_{2}(X)$ is equipped with the following norm. For $a \in V_{2}(X)$,

$$
\|a\|_{V_{2}}=\inf \left\{C_{1} C_{2}:(1) \text { and (2) hold }\right\} \text {. }
$$

For the moment, let $G$ be a locally compact group. Let $A(G)=L^{2} * L^{2}(G)$ be the Fourier algebra of $G$ and $B(G)$ the Fourier-Stieltjes algebra, that is, the space of coefficients of unitary representations of $G$. Denote by $B_{2}(G)$ the Herz-Schur multiplier algebra, defined as follows. A continuous function $f \in B_{2}(G)$ if the invariant kernel $f\left(y^{-1} x\right)$ belongs to $V_{2}(G)$. The algebra $B_{2}(G)$ is equipped with the norm \|\|$_{B_{2}}$ induced from $V_{2}(G)$. Observe that $B(G) \subset B_{2}(G)$ and \|\|$_{B_{2}} \leq\|\|_{B}$. Denote by $A_{c}(G)$ the subspace of $A(G)$ of compactly supported function, and by $B_{\lambda}(G)$ the closure of the space of coefficients of the left regular representation in the topology on uniform convergence 
on compact sets boundedly in the norm of $B(G)$. Recall that $G$ is amenable if $B_{\lambda}(G)=B(G)$, or equivalently, $1 \in B_{\lambda}(G)$. That is, if for some $C>0$ there exists a net $v_{i} \in A_{c}(G)$ such that $\left\|v_{i}\right\|_{B\langle G\rangle} \leq C$ and $v_{i} \rightarrow 1$ uniformly on compact sets.

Definition. A function $f$ on $G$ belongs to $B_{2, \lambda}(G)$ if there exists a net $f_{i} \in$ $A_{C}(G)$ such that $f_{i} \rightarrow f$ uniformly on compact sets and $\left\|f_{i}\right\|_{B_{2}} \leq C$ for some $C>0$.

A locally compact $G$ is weakly amenable if $B_{2, \lambda}(G)=B_{2}(G)$ or equivalently, $1 \in B_{2, \lambda}(G)$, i.e., there exists a net $u_{i} \in A_{c}(G)$ such that $\left\|u_{i}\right\|_{B_{2}} \leq C$ and $u_{i} \rightarrow 1$ uniformly on compact sets. Let $\Lambda_{G}$ be the infimum of all such constants $C$ for all nets as above.

We now focus attention on the multiplier space $V_{2}(X)$ in the case where $X$ is a tree. Let $\omega$ be an end (i.e., an infinite ray originating at some reference vertex $o$ of $X)$. Let $d(x, y)$ be the natural distance on $X$. For $n \in \mathbb{N}$, let $E_{n}=\{(x, y) \in X \times X: d(x, y)=n\}$, and denote by $\chi_{n}=\chi_{E_{n}}$ the characteristic function of $E_{n} \subset X \times X$.

For $x \in X$, there exists a unique geodesic ray $\gamma$ starting at $x$ that coincides with $\omega$ except for a finite number of vertices [Ca]. It is called the geodesic from $x$ to $\omega$. The derivation $T_{\omega}$ along $\omega$ is defined by $T(x)=T_{\omega}(x)=x^{+}$, where $x^{+} \in \gamma$ and $d\left(x, x^{+}\right)=1$. In the context of Herz-Schur multipliers, it is appropriate to regard $T_{\omega}$ as a mapping from $X$ to $\ell^{2}(X): T_{\omega}(x)=\delta_{x^{+}}$.

For the sake of brevity, in the remainder of this section we write the $\ell^{2}(X)$ inner product $\left\langle\delta_{x}, \delta_{y}\right\rangle$ as $\langle x, y\rangle$.

Proposition 2.1. $\chi_{n} \in V_{2}(X)$ and $\left\|\chi_{n}\right\|_{V_{2}} \leq 2 n$, for every $n \in \mathbb{N}$.

Proof. Observe that, for any two distinct vertices $x, y$, either they lie in the same geodesic towards $\omega$, or they lie in two different geodesics that merge after finitely many steps. It follows easily that the functions $\varphi_{2 n}=\chi_{0}+\chi_{2}+$ $\cdots+\chi_{2 n}$ and $\varphi_{2 n+1}=\chi_{1}+\chi_{3}+\cdots+\chi_{2 n+1}$ satisfy the identity $\varphi_{n}(x, y)=$ $\sum_{k=0}^{n}\left\langle T^{n-k}(x), T^{k}(y)\right\rangle$ for every $n \in \mathbb{N}$. Clearly, $\left\|\varphi_{n}\right\|_{V_{2}(X)} \leq n+1$. But $\chi_{n}=\varphi_{n}-\varphi_{n-2}$. Therefore $\left\|\chi_{n}\right\|_{V_{2}(X)} \leq 2 n$. (A different, but similar, proof was given in [PS].)

\section{Free products With amalgamation aNd their LENGTH FUNCTIONS}

We now turn our attention to amalgams. Let $I$ be a (not necessarily finite) index set. We recall the definition of the amalgamated product $G={ }^{A} G_{i}$ of a family of groups $\left(G_{i}\right)_{i \in I}$ over a group $A$ that embeds into each $G_{i}$ via an injective homomorphism $\lambda_{i}$ (see, for instance, [Se]). Let $G$ be a group and $\rho_{i}: G_{i} \rightarrow G, i \in I$, a family of homomorphisms such that $\rho_{i} \circ \lambda_{i}=\rho_{j} \circ \lambda_{j}$ for all $i, j \in I$. Then $G=*_{A} G_{i}$ if, for every group $H$ and for every family of homomorphisms $\tau_{i}: G_{i} \rightarrow H, i \in I$, with the property that $\tau_{i} \circ \lambda_{i}=\tau_{j} \circ \lambda_{j}$ for all $i, j \in I$, there exists a homomorphism $\rho: G \rightarrow H$ such that $\rho \circ \rho_{i}=\tau_{i}$ for all $i$. In other words, $G=*_{A} G_{i}$ if the following diagram commutes for every $i, j \in I$ : 


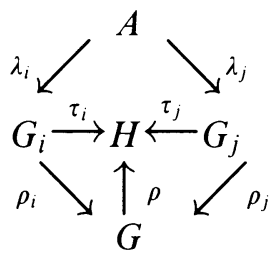

Observe that, in the particular case where $A$ is trivial, $G=* G_{i}$ is the free product of the $G_{i}$ 's.

If $G_{1}, G_{2}$ are topological groups, then their topological free product with amalgamation, with respect to a common closed subgroup $A$, is defined by replacing all the homomorphisms in the definition of amalgamated product by continuous homomorphisms [Grl]. Many interesting properties of topological amalgamated products can be found in [Gr2]; see also [Al2].

It was shown in [A12] that if the topological amalgamated product $G=G_{1} *_{A}$ $G_{2}$ is locally compact, then $A, G_{1}$, and $G_{2}$ are open in $G$. Conversely, if $A$ is an open subgroup of the locally compact groups $G_{1}, G_{2}$, then the amalgam $G=G_{1} *_{A} G_{2}$ admits a locally compact topology inherited in which $A, G_{1}$, and $G_{2}$ are open (see, for instance, [Al1] and references therein).

In the sequel, when referring to free products with amalgamation, we shall always mean topological free products with amalgamation. Of course, this remark is unnecessary when dealing with amalgams of discrete groups (for instance, with free groups), but it is important in the next example. There are many interesting examples of amalgams besides free groups. See, for instance, [Se]. We limit attention to the only explicit example that will be relevant later.

Example. Let $k$ be a field with a discrete valuation $\nu$ (for example, $k=\mathbb{Q}_{p}$, the $p$-adic field), and let $\mathbb{O}_{k}=\{x \in k: \nu(x) \geq 0\}$ be its ring of integers. Denote by $\mathscr{P}_{k}=\{x \in k: \nu(x)>0\}$ the unique maximal ideal in $\mathbb{O}_{k}$. Set $\Gamma=\mathrm{SL}_{2}\left(\mathbb{O}_{k}\right)$ and $A=\left\{\left(\begin{array}{ll}a & b \\ c & d\end{array}\right) \in \Gamma: c \in \mathscr{P}_{k}\right\}$. Then [Ih] $\mathrm{SL}_{2}(k)=\Gamma *_{A} \Gamma$ (see also [Se]).

It is well known that amalgams are deeply connected with trees. Indeed, the elements of ${ }_{A_{A}} G_{i}$ can be represented as words in the $G_{i}$ 's. More precisely, for every $i \in I$, let $S_{i}$ be a complete collection of right coset representatives of $A \backslash G_{i}$, with $e \in S_{i}$. That is, the map $(a, s) \rightarrow$ as is a bijection between $A \times S_{i}$ and $G_{i}$ such that the image of $A \times\left(S_{i} \backslash\{e\}\right)$ is $G_{i} \backslash A$. The reduced words are the finite sequences $w=\left(a, s_{1}, \ldots, s_{n}\right)$ with $a \in A, s_{k} \in S_{j_{k}}, s_{k} \neq e \forall k$, $j_{k} \neq j_{k+1} \forall k$. The bijection between elements $g \in G=*_{A} G_{i}$ and reduced words $w=\left(a, s_{1}, \ldots, s_{n}\right)$ is given by $g=j(a) j_{i_{1}}\left(s_{1}\right) \cdots j_{i_{n}}\left(s_{n}\right)$.

Moreover, if $G=G_{1} *_{A} G_{2}$, there exists a tree $X$ (and only one, up to isomorphism) on which $G$ acts with an inversion. The action is isometric, and the fundamental domains are the segments, that is, the pairs of neighbors $p_{1}, p_{2}$, together with the edge $\tau=\left[p_{1}, p_{2}\right] . G_{i}$ is the stabilizer of $p_{i}$ in $G$ $(i=1,2)$, and $A$ is the stabilizer of $\tau$. (For proofs, see [Se].)

The integer $n$ in the expression of the reduced word associated with $g$ is called the length of $g$ and is denoted by $\|g\|$. Note that $\|g\|=0$ if and only if $g \in A$, and $\|g\|=1$ if and only if $g \in G_{i} \backslash A$. It is easy to exhibit a tree $X$ on which $G=G_{1} *_{A} G_{2}$ acts. 
Indeed,

$$
\begin{aligned}
\operatorname{vert}(X) & =\left(G / G_{1}\right) \cup\left(G / G_{2}\right) & & \text { (disjoint union), } \\
\operatorname{edge}(X) & =G / A & & \text { (nonoriented edges), }
\end{aligned}
$$

and the cosets $g G_{1}$ and $g G_{2}$ are connected by the edge $g A$. In this realization, the segment consisting of the vertices $G_{1}, G_{2}$, and the edge $A$ is a fundamental domain for the natural action of $G$ (by translation). It is not difficult to verify that $X$ is a tree and that the length function $\|g\|$ is related to the distance on $X$ by $2\|g\|=d\left(g G_{1}, G_{1}\right)+d\left(g G_{2}, G_{2}\right)+\chi_{A}(g)-1$, where $\chi_{A}$ is the characteristic function of $A$.

If $G$ is an amalgamated product of more than two factors, however, this construction yields a graph $\Gamma$ that is not a tree. For instance, if $G=*_{A} G_{i}$ with $i=1, \ldots, n$, then $\Gamma$ contains subgraphs isomorphic to the complete graph on $n$ vertices (all the $G_{i}$ 's form pairwise contiguous vertices, connected by copies of the edge $A$ ). Therefore, in general, we make use of another tree $T$ on which the amalgam $G=*_{A} G_{i}$ acts isometrically. This tree can be thought of as the barycentric subdivision of $X$. Its construction is due to I. M. Chiswell [Ch] (see also [Se]). The same realization of the tree of an amalgamated product has been considered in [PW] to obtain a local limit theorem on amalgams and in [FP2] to prove irreducibility of certain spherical representations upon restriction to a lattice.

The vertices of $T$ are all the cosets $g G_{i}$ and $g A$, where $g \in G$ and $i \in I$. The vertex $A$ is regarded as the central vertex or root. The (unoriented) edges are the pairs $\left[g A, g G_{i}\right], g \in G, i \in I$. Denote by $l_{\circ}$ the length function of $G$ corresponding to the rooted tree $(T, A)$. Namely, for $g \in G, l_{\circ}(g)$ is the distance between $A$ and $g A$ in $T$.

Lemma $3.1[\mathrm{Ch}]$. The natural length \|\| on $G$ as a free product with amalgamation is related to Chiswell's length function $l_{\circ}$ by

$$
l_{\circ}(g)=2\|g\| \text {. }
$$

Now we put together these preliminaries with the contents of $\S 1$. The first part of the next proposition follows also from Theorem 1 of [Pi] (see also [Bo2]).

Proposition 3.2. Let $G={ }_{A} G_{i}$. Then

(i) for each $t>0, \rho_{t}(g)=e^{-t\|g\|}$ is positive definite on $G$;

(ii) if $\chi_{n}$ denotes the characteristic function of $E_{n}=\{g \in G$; $\|g\|=n\}$, then $\chi_{n} \in B_{2}(G)$ and $\left\|\chi_{n}\right\|_{B_{2}} \leq 4 n$, for every $n \in \mathbb{N}$.

Proof. Since the action of $G$ on the tree $T$ is isometric, Lemma 3.1 yields, for $g, h \in G$

$$
2\left\|g^{-1} h\right\|=\operatorname{dist}(h A, g A) .
$$

But the distance $d($,$) is a negative kernel of T$, as shown in [Ha1]. (More precisely, the latter reference deals with free groups, but the argument depends only on the geometric of their Cayley graphs, which are trees even homogeneity degree. The same argument holds also for trees with odd homogeneity.) Now (i) follows from Schoenberg's theorem [dHV, Chapter 4, Theorem 12]. Proposition 2.1 yields part (ii). 


\section{WEAK AMENABILITY OF TOPOLOGICAL FREE GROUPS WITH AMALGAMATION}

In this final section, we prove that the amalgamated product of a family of amenable groups $G_{i}$ over a common compact open subgroup $A$ is weakly amenable, with Herz-Schur constant $\Lambda_{G}=1$. This extends a result proved in [Pi] under the assumption that the indices $\left[G_{i}: A\right]$ are bounded. As we have seen in the previous section, the typical example to which our theorem applies is $\mathrm{SL}_{2}\left(\mathbb{Q}_{p}\right)$ (see also [Sz]).

It was observed in [FP1, Corollary VIII.1.6] that free groups have approximate identities bounded in the norm of multipliers of the Fourier algebra, and that the infimum of these bounds is 1 . In particular, this yields the same result for the group $\mathrm{SL}_{2}(\mathbb{Z})$, which contains a finite-index free subgroup (of course, this also follows from [Pi], because $\mathrm{SL}_{2}(\mathbb{Z})$ is an amalgam over a finite subgroup, or from [dCH, EW]). It has been proved in $[\mathrm{CoH}]$ that lattices in semisimple Lie groups of rank one are weakly amenable, although $\Lambda_{G}>1$, in general. But semisimple Lie groups of rank larger than one are not weakly amenable [Ha2]. As a consequence $[\mathrm{CoH}]$, lattices therein provide examples of discrete groups that are not weakly amenable.

We need a preliminary lemma, whose elementary proof is left to the reader.

Lemma 4.1. Let $A$ be a compact subgroup in a locally compact group $G$, and let $u$ be a positive definite function on $G$ such that $u(e)=1$ and $\int_{A} u(a) d a \neq 0$. Let $u(x)=\langle\pi(a) \xi, \xi\rangle$ and $c=\left\|\int_{A} \pi(a) \xi d a\right\|$. Then the function $v$ on $G$, defined by

$$
v(x)=c^{-2} \int_{A} \int_{A} u\left(h^{-1} x g\right) d h d g,
$$

is also positive definite, satisfies $v(e)=1$, and is left and right invariant under $A$.

Theorem 4.2. Let $\left\{G_{i}, i \in I\right\}$ be a family of amenable locally compact groups, and let $A$ be an open compact subgroup of $G_{i}$ for each $i \in I$. Then $G={ }_{A} G_{i}$ is a weakly amenable group, with Herz-Schur multiplier constant $\Lambda_{G}=1$.

Proof. As the groups $G_{i}$ are amenable and $A$ is a compact subgroup, Lemma 4.1 shows that there exists a net of positive definite function $\left(\varphi_{j}^{i}\right)_{j \in J}$ with compact support on $G_{i}$ such that $\varphi_{j}^{i}(a)=1$ for $a \in A ; \varphi_{j}^{i}(a x)=\varphi_{j}^{i}(x a)=\varphi_{j}^{i}(x)$ for $x \in G_{i}, a \in A, i \in I, j \in J$; and $\varphi_{j}^{i} \rightarrow 1$ uniformly on compact sets in $G_{i}$. Note that $\left|\varphi_{j}^{i}(x)\right| \leq 1$ for every $x \in G_{i}$, as $\varphi_{j}^{i}$ is positive definite, and therefore $\left\|\varphi_{j}^{i}\right\|_{B_{2}} \leq\left\|\varphi_{j}^{i}\right\|_{B}=1$. Now, as in [Pi], we form the amalgamated free product function on $G$ defined by $\varphi_{j}=*_{i \in I} \varphi_{j}^{i}$. By Theorem 1 of $[\mathrm{Pi}], \varphi_{j}$ is also positive definite on $G=*_{A} G_{i}$. Moreover, $\varphi_{j}$ converges to 1 uniformly on compact subsets of $G$, but of course it is not compactly supported. We need to approximate $\varphi_{j}$ with compactly supported functions, uniformly on compact sets and boundedly in the norm of $B_{2}(G)$. However, the index set $I$ may not be finite and the groups $G_{i}$ are not necessarily compact. The former obstacle is easy to overcome. Consider the net of all finite subsets $I_{\alpha}$ of $I$, and form $\varphi_{j}^{\alpha}=*_{i \in I_{\alpha}} \varphi_{j}^{i}$ on $G_{\alpha}=*_{i \in I_{\alpha}} G_{i}$. Regard $\left\{\varphi_{i}^{\alpha}\right\}$ as a net of functions in $G$. Then this net converges to 1 uniformly on compact sets. However, contrary to [Pi], we still need to truncate $\varphi_{j}^{\alpha}$, because the $G_{i}$ 's are not compact. On the other hand, truncating $\varphi_{j}^{\alpha}$ forces its $B_{2}$-norm 
to blow up. Therefore we need to mollify the truncations with a smoothly decaying multiplier. This multiplier is constructed as follows. For $0<r<1$, let $f_{r, n}(g)=\psi(g) \sum_{k=0}^{n} \chi_{k}(g)=r^{\|g\|} \sum_{k=0}^{n} \chi_{k}(g)$, where, as in Proposition 2.2(ii), $\chi_{k}$ is the characteristic function of the words of length $k$ in $G$. Observe that $f_{r, n}(g)=r\|g\|-\sum_{k>n} r^{k} \chi_{k}(g)$. Since $\left\|\chi_{k}\right\|_{B_{2}} \leq 4 k$ by Proposition 2.2(ii), for all $\varepsilon>0$ and $0<r<1$ there exists $n=n(r)$ such that $\left\|\sum_{k>n} r^{k} \chi_{k}(g)\right\|_{B_{2}} \leq \varepsilon$. Now choose $\varepsilon=\varepsilon(r) \rightarrow 0$ as $r \rightarrow 1$. Then there exists $n=n(r)$ such that $\left\|f_{r, n\langle r\rangle}\right\|_{B_{2}}<1+\varepsilon(r)$. Now the function $\omega_{j, r}=\varphi_{j} f_{r, n(r)}$ has compact support and $\left\|\omega_{j, r}\right\|_{B_{2}}<1+\varepsilon(r)$, with $\varepsilon(r) \rightarrow 0$ as $r \rightarrow 1$. Thus, when $r \rightarrow 1$ and $j \rightarrow \infty, \omega_{j, r} \rightarrow 1$ uniformly on compact sets in $G$, boundedly in the $B_{2}$ norm. Therefore $G$ is weakly amenable. Moreover, $\Lambda_{G}=1$, as $\varepsilon(r) \rightarrow 0$ for $r \rightarrow 1$.

It is a consequence of the results of [Pi] that $\mathrm{SL}_{2}\left(\mathbb{Q}_{p}\right)$ has approximate identities bounded in $\mathscr{M}(A)$. The previous theorem improves this result (see also [Sz]):

Corollary 4.3. $\mathrm{SL}_{2}\left(\mathbb{Q}_{p}\right)$ is weakly amenable.

Proof. It is enough to recall the example of $\S 3$, namely,

$$
\operatorname{SL}\left(2, \mathbb{Q}_{p}\right)=\operatorname{SL}\left(2, \mathbb{Z}_{p}\right) * \Gamma_{p} \operatorname{SL}\left(2, \mathbb{Z}_{p}\right),
$$

where $\mathbb{Z}_{p}$ is the ring of $p$-adic integers, which is compact, and

$$
\Gamma_{p}=\left\{\left(\begin{array}{ll}
a & b \\
c & d
\end{array}\right) \in \operatorname{SL}\left(2, \mathbb{Z}_{p}\right): c \equiv 0(\bmod p)\right\}
$$

is an open compact subgroup of $\operatorname{SL}\left(2, \mathbb{Z}_{p}\right)$.

As in [Pi], Theorem 4.2 yields a result about convolution operators on $L^{p}(G)$ and its dual space. The proof is the same as in [Pi].

Corollary 4.4. If $G$ is as in Theorem 4.1, then $C V_{p}(G)=A_{p}(G)^{*}$ for $1<p<$ $\infty$.

\section{REFERENCES}

[Al1] R. Alperin, Locally compact group acting on trees, Pacific J. Math. 1 (1982), 23-31.

[A12] Locally compact groups acting on trees and property T, Monatsh. Math. 93 (1982), 261-265.

[Bo] M. Bożejko, Positive definite bounded matrices and a characterization of amenable groups, Proc. Amer. Math. Soc. 95 (1985), 357-360.

[Bo2] _ Uniformly bounded representations of free groups, J. Reine Angew. Math. 377 (1987), 170-187.

[BF] M. Bożejko and G. Fendler, Herz-Schur multipliers and completely bounded multipliers of the Fourier algebra of a locally compact group, Boll. Un. Mat. Ital. (6) 6 (1984), 1275-1280.

[Ca] P. Cartier, Fonctions harmoniques sur un arbre, Sympos. Math., vol. 9, Academic Press, 1972, pp. 203-270.

[Ch] I. M. Chiswell, Length functions and free products of groups, Proc. London Math. Soc. (3) 43 (1981), 42-58.

[Co] M. Cowling, Sur les coefficients des représentations unitaires des groupes de Lie simples, Lecture Notes in Math., vol. 739, Springer-Verlag, Berlin and New York, 1979, pp. 132-178.

[CoH] M. Cowling and U. Haagerup, Completely bounded multipliers of the Fourier algebra of a simple Lie group of real rank one, Invent. Math. 96 (1989), 507-549. 
[dCH] J. de Canniere and U. Haagerup, Multipliers of the Fourier algebra of some simple Lie groups and their discrete subgroups, Amer. J. Math. 107 (1984), 455-500.

[dHV] P. de la Harpe and A. Valette, La proprieté (T) de Kazhdan pour les groupes localement compacts, Astérisque 175 (1989).

[Di] J. Dixmier, Les $C^{*}$-algèbres et leurs Représentations, Gauthier-Villars, Paris, 1969.

[EW] M. Enomoto and Y. Watatani, Reduced group $C^{*}$-algebras with the metric approximation property by positive maps, Proc. Japan Acad. Ser. A 63 (1987), 304-306.

[FP1] A. Figà-Talamanca an dM.A. Picardello, Harmonic Analysis on Free Groups, Lecture Notes in Pure and Appl. Math., vol. 87, M. Dekker, New York, 1983.

[FP2] _ Restriction of spherical representations of $P G L_{2}\left(\mathbb{Q}_{p}\right)$ to a discrete subgroup, Proc. Amer. Math. Soc. 91 (1984), 405-408.

[Gr1] M. J. Graev, Free topological groups, Izv. Akad. Nauk 114 (1950), 343-354.

[Gr2] _ Theory of topological groups, norms and metrics on groups, complete groups, free topological groups, Uspekhi Mat. Nauk 2 (1950), 3-56.

[Ha1] U. Haagerup, An example of a non-nuclear $C^{*}$-algebra which has the metric approximation property, Invent. Math. 50 (1979), 279-293.

[Ha2] $\_$, Group $C^{*}$-algebras without the completely bounded approximation property, preprint, 1986.

[Ih] Y. Ihara, On discrete subgroups of the two by two projective linear group over p-adic fields, J. Math. Soc. Japan. 18 (1966), 219-235.

[He] C. Herz, Une generalization de la notion de transformée de Fourier-Stieltjes, Ann. Inst. Fourier (Grenoble) 24 (1974), 145-157.

[Lo] V. Losert, Properties of the Fourier algebra that are equivalent to amenability, Proc. Amer. Math. Soc. 92 (1984), 347-354.

[Pi] M. A. Picardello, Positive definite functions and $L^{p}$ convolution operators on amalgams, Pacific J. Math. 123 (1986), 209-221.

[PW] M. A. Picardello and W. Woess, Random walks on amalgams, Monatsh. Math. 100 (1985), 21-33.

[PS] T. Pytlik and R. Szwarc, An analytic family of uniformly bounded representations of free groups, Acta Math. 187 (1986), 287-309.

[Se] J.-P. Serre, Trees, Springer-Verlag, New York and Berlin, 1980.

[Sz] R. Szwarc, Groups acting on trees and approximation properties of the Fourier algebra, preprint.

Mathematical Institute, University of Wroclaw, Pl. Grunwaldzki 2/4, 50-384 WROCLAW, Poland

E-mail address: porembsk@plwrtu11.bitnet

Dipartimento di Matematica, Università di Roma "Tor Vergata", Via Ricerca ScienTIFICA 00133 ROMA, ITALY

E-mail address: picard@itcaspur.bitnet, picard@vaxtvm.infn.it 